Methods Patients were recruited to the program either by selfreferral or by ILD clinicians. Seven participants were recruited. The 6-week PR program consisted of 60 minutes exercise and 30 minutes education with the emphasis on strength training over endurance. Focus group interviews were used to collect qualitative data and analysed using an inductive approach utiilising thematic analysis as a method.

Results 7 patients completed the programme (5M:2F, mean age 73.4). Initial qualitative analysis demonstrates psychological benefit from the sharing of disease experiences, prioritising exercise as a means of management, empowerment and understanding. 6MWT and Kings Brief ILD questionnaire showed no significant change pre and post PR, see Table 1 . These quantitative results do not reflect patient perceptions of improved functional status.

Conclusion Patients perceptions were positive regarding the content and impact it had on their education needs concerning their disease. The predominant perceived benefit was that of the comradery found in sharing experiences with other ILD patients. No firm conclusions can be drawn from this study regarding the effectiveness of PR for patients with ILD due to small numbers. It is unclear whether the tools used to assess functional and health measures are suitable to detect changes in outcomes post PR or whether the effects of an ILD PR programme are predominantly that of disease management and education, explaining the disparity observed between quantitative and qualitative outcomes. Further appropriately powered controlled studies could examine the impact of PR and in particular that of PR education specifically for ILD patients, as supported by Holland et al. (2015).

$\begin{aligned} & \text { Abstract P280 Table } 1 \\
& \text { for 6MWD and Kings ILD questionnaire. }\end{aligned}$
\begin{tabular}{llll}
\hline Six Minute Walk & Kings ILD Questionnaire & & \\
Distance & $\begin{array}{l}\text { Breathlessness \& } \\
\text { Activities }\end{array}$ & Psychological & Chest \\
& +0.5 & -1 & -0.5 \\
\hline $12 \mathrm{~m}$ & & & Symptoms \\
\hline
\end{tabular}

\section{P281 ANNUAL CHANGE IN PULMONARY FUNCTION IN ASBESTOSIS}

S Clarke, J Hoyle. North Manchester General Hospital, Manchester, UK

\subsection{6/thoraxinl-2016-209333.424}

Introduction Asbestosis is commonly considered to be associated with slowly progressive pulmonary fibrosis. However, there is limited recent data to support this opinion. We set out to analyse the change in pulmonary function test (PFT) over time in a cohort of outpatients with asbestosis.

Methods Patients were identified retrospectively from a pool of clinic patients who had consented to participate in research. The diagnosis of asbestosis had been made on CT findings, history of asbestos exposure, exclusion of other causes of interstitial lung disease and agreement at occupational MDT. The PFT data from tests closest to the time of initial diagnosis were compared to the most recent PFT results. Parameters assessed were $\mathrm{FEV}_{1}, \mathrm{FVC}$, VC, TLC and KCO. The values were expressed as percentage predicted to ensure adjustment for age, weight etc. Annual change was calculated by dividing the total change by the number of years elapsed between PFT. Smoking status was also documented.

Results 57 patients were identified with a diagnosis of asbestosis. 9 had only had 1 set of PFT (awaiting follow-up) and hence were excluded leaving 48 patients. The mean time difference between PFT was 3.0 years (range $0.2-6.1$ years). In 21 cases TLC had not been measured in one or both PFT and in $10 \mathrm{KCO}$ had not been measured.

Conclusions As expected baseline $\mathrm{FEV}_{1}$ decreased with increased smoking exposure. The other parameters at baseline were lower in those with the highest smoking exposure with the exception of TLC which was more varied. The greatest rate of change was seen in $\mathrm{KCO} \%$ predicted, consistent with previous research. Unexpectedly the groups demonstrating the maximal decline in KCO were those with a low $(<20$ pack year) smoking history (7.2\% annual decline) followed by lifelong non-smokers $(3.9 \%$ annual decline). Those with the heaviest smoking history showed a lower rate of decline in all parameters compared to both nonsmokers and the population as a whole. KCO and TLC were not performed in those with the most severe disease due to breathlessness, thus these results are likely to be an under-estimate of lung function changes.

\section{P282 COMPARITIVE USE OF NHANES III, ECCS AND GLI PREDICTION EQUATIONS IN DETERMINING SPIROMETRIC INDICES AND SUITABILITY FOR ANTI- FIBROTIC THERAPY IN PATIENTS WITH IDIOPATHIC PULMONARY FIBROSIS}

I Cliff, A Ali, M Spiteri, H Stone. Department of Respiratory Medicine, University Hospitals of North Midlands, Royal Stoke University Hospital, Stoke on Trent, UK

\subsection{6/thoraxjnl-2016-209333.425}

Introduction Prediction equations are used to assess disease severity and prognosis in respiratory disease; globally most laboratories utilise ECCS or NHANES III equations. The Global Lung Initiative (GLI) produced reference ranges for spirometry that are multi-ethnic and applicable for patients upto the age of 95. The choice of which equation to use becomes crucial in idiopathic pulmonary fibrosis [IPF] patients, in whom prescription of currently available anti-fibrotic agents, Nintedanib and Pirfenidone is dependent on a forced vital capacity [FVC] between 50 and $80 \%$ of predicted in England and Wales (Scotland only restriction is FVC above $80 \%$ predicted).

Methods Spirometric data recorded on 132 IPF patients were extracted from our BTS ILD Registry database. Values for FVC\% predicted were calculated using the ECCS, NHANES III and GLI equations and compared to determine patient eligibility for antifibrotic treatment in line with published NICE Guidance.

Results Data on 132 consecutive patients is presented in Table 1. This demonstrates the FVC\% predicted when the 3 separate equations are used. At our centre, where ECCS is routinely used to calculate $\mathrm{FVC} \%$ predicted, 62 patients (47\%) of patients had an FVC above the upper limit of the treatment threshold of $80 \%$. Of this group, 8 had evidence of more than 25\% emphysematous change on their HRCT scans. 